\title{
Design and Evaluation of joint resistance in SSC Rutherford type cable splices for Torus magnet for the Jefferson Lab $12 \mathrm{GeV}$ Upgrade
}

Probir K. Ghoshal, Senior Member, IEEE, Ruben J. Fair, Senior Member, IEEE, Damian Hampshire, Virginia Hagen-Gates, David Kashy, Robert Legg, Renuka Rajput-Ghoshal, Yeekin Tsui

Abstract - The Hall B 3.6 T superconducting torus magnet is being designed and built as part of the Jefferson Lab $12 \mathrm{GeV}$ upgrade. The magnet consists of 6 trapezoidal coils connected in series with an operating current of $3770 \mathrm{~A}$. The magnet and the joints (or splices) connecting the coils are all conduction cooled by supercritical $4.6 \mathrm{~K}$ helium. This paper evaluates the stability requirements for the splices made between SSC Rutherford type cables. The basis for design and tests performed to evaluate the performance of the splices under varying incident magnetic fields are discussed along with test results.

Index Terms - SSC Cable, conduction cooled, superconducting magnet, torus coil, splices, joints, transport current, magnetic field.

\section{INTRODUCTION}

$\mathrm{O}^{2}$ ne of the main challenges with the Jefferson Lab $12 \mathrm{GeV}$ upgrade is the size and complexity of the torus magnet system which forms a part of the CLAS12 spectrometer in HALL B [1].

The torus magnet consists of six superconducting coils which are connected and arranged (Fig 1) to produce a toroidal magnetic field. Torus coil parameters are shown in Table 1. These double pancake coils have 234 total turns, are vacuum impregnated with epoxy, assembled into an aluminum case and are indirectly cooled by supercritical helium gas, forming what is referred to as the coil cold mass (CCM). Each coil within the aluminum case is conduction cooled via a series of thin copper sheets wrapped round the coil winding pack and soldered to the helium cooling tube. Each CCM is surrounded by a nitrogen shield located between the vacuum vessel walls and the coil case [2]. All six coils are electrically connected in series using superconducting conductor extending from the coils that are connected to one another at soldered joints. The slightly resistive joints are cooled by mounting them directly onto the local supercritical helium heat exchangers called re-coolers.

The individual coils are wound with surplus outer dipole SSC cable (see parameters in Table II), with $2 \times 18$ strands having key-stoned dimensions of $1.053 \mathrm{~mm} \times 1.259 \mathrm{~mm} \times$

Manuscript received Oct 20, 2015. Authored by Jefferson Science Associates, LLC under U.S. DOE Contract No. DE-AC05-06OR23177. The U.S. Government retains a non-exclusive, paid-up, irrevocable, world-wide license to publish or reproduce this manuscript for U.S. Government purposes.

Probir K. Ghoshal, Ruben J. Fair, David Kashy, Robert Legg, Renuka Rajput-Ghoshal are all with Jefferson Lab, Newport News, VA (USA), 23606. Virginia Hagen-Gates is with University of Houston, Houston, TX (USA), Damian Hampshire and, Yeekin Tsui is with Department of Physics, Durham University, Durham (UK). Phone: 757-269-6702; e-mail: ghoshal@jlab.org
$11.68 \mathrm{~mm}$. The cable is further stabilized by soldering into an extruded OFHC copper channel with $\mathrm{Sn}_{60} \mathrm{~Pb}_{40}$ solder - as shown in Fig. 2.

The resistance of the lap splice at each joint is designed for operation at 4.6 K. JLab set the requirement for each splice to have a power loss no higher than $100 \mathrm{~mW}$. For operation using $3770 \mathrm{~A}$ at $4.6 \mathrm{~K}$ the splice resistance can therefore be no higher than $7 \times 10^{-9} \Omega$, The splices must also operate in magnetic fields up to $0.3 \mathrm{~T}$ at these currents and temperatures. The splice is cooled via copper braids soldered to the recoolers. The splice design was also constrained by the available space around the re-cooler units that are inside the downstream cold beams that help position the coils in the hexagonal array. Another requirement of the joint design was adequate electrical isolation from ground that could stand off $2,500 \mathrm{~V}$ in a vacuum

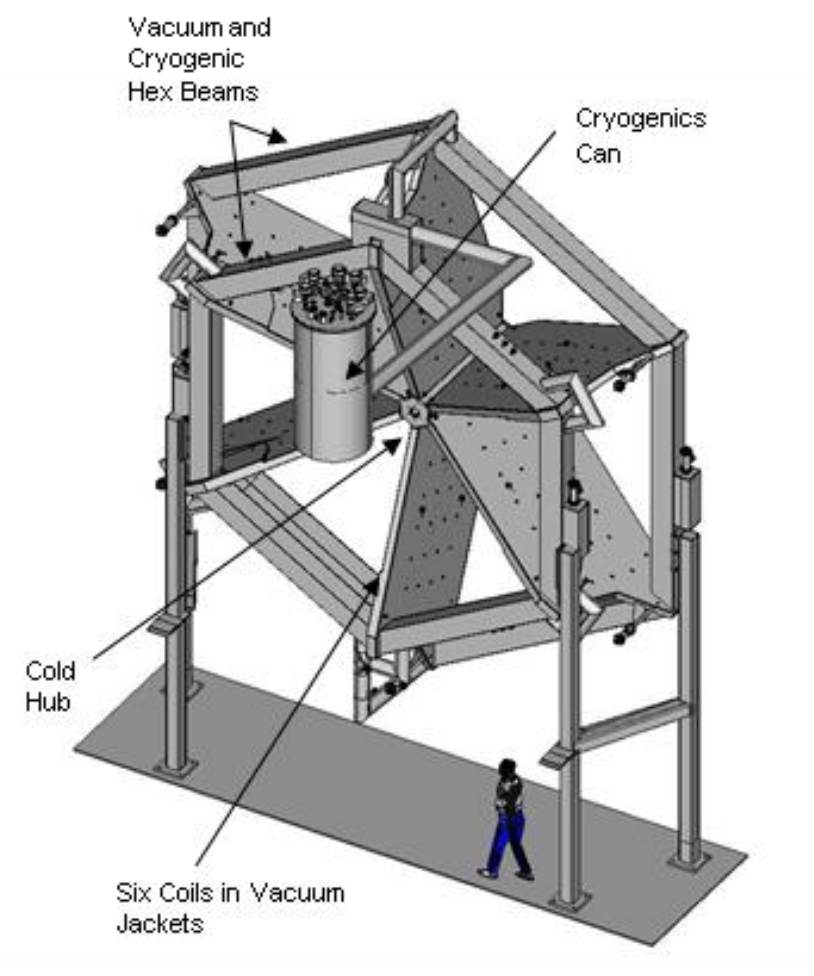

Fig. 1: CLAS12 torus magnet overview.

Splice Mechanical Design - Reliable low resistance electrical joints are vital to the safe operation of the torus magnet. The structure of the typical single splice we developed starts with intimate contact between the splicing superconducting cables to minimize contact resistance. This construction, with the inside of the copper channels surrounding the cable, facing each other is shown in Fig. 3. 
The second structural feature adds additional stabilizing copper over and above the copper channel in the form of a rectangular section of copper which we discuss below.

TABLE I: TORUS COIL PARAMETERS

\begin{tabular}{lcc}
\hline \multicolumn{1}{c}{ Parameter } & Unit & Value \\
\hline Peak operating current & $\mathrm{A}$ & 3770 \\
Coil peak field & $\mathrm{T}$ & 3.58 \\
Operating temperature (nominal) & $\mathrm{K}$ & 4.6 \\
Number of coils & {$[\mathrm{]}$} & 6 \\
Total number of turns/coil & {$[\mathrm{]}$} & $2 \times 117$ \\
Superconducting cable dimensions & $\mathrm{mm} \mathrm{x} \mathrm{mm}$ & $2.5 \times 20$ \\
NbTi strand bare diameter & $\mathrm{mm}$ & 0.648 \\
Number of strands in the cable & {$[$ ] } & 36 \\
Cu:Sc ratio (strand) & {$[$ ] } & 1.8 \\
Total stored energy & $\mathrm{MJ}$ & 14.2 \\
Inductance & $\mathrm{H}$ & 2 \\
\hline \hline
\end{tabular}

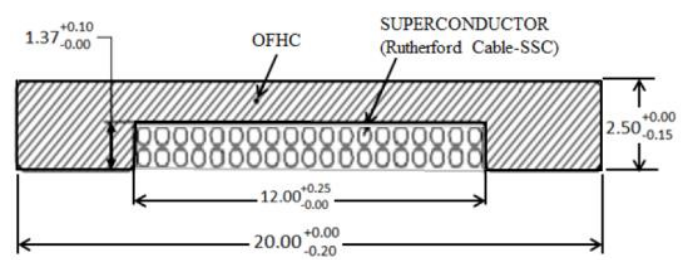

Fig. 2: Torus conductor - SSC outer cable soldered into copper channel (Dimensions in $\mathrm{mm}$ )

TABLE II: TORUS CONDUCTOR SPECIFICATION

\begin{tabular}{lc}
\hline \hline Parameter & Details \\
\hline Rutherford type of cable (Superconductor) & $\mathrm{NbTi}$ \\
Conductor material (NbTi $+\mathrm{Cu})$ & $\mathrm{Cu}-(\mathrm{NbTi})$ in Cu Channel \\
Number of strands in the cable & 36 \\
Number of NbTi filaments in each strand & 4600 \\
Strand bare diameter (mm) & 0.648 \\
Copper to non-Copper ratio & 1.8 \\
Twist pitch (mm) & 15 \\
Conductor size (bare) (mm x mm) & $20 \times 2.5$ \\
Conductor size (insulated) (mm x mm) & $20.2 \times 2.7$ \\
Short sample current at 4.22 K, 5 T (kA) & 11 \\
RRR Cu (Cu-NbTi) - Strand & 100 \\
RRR Cu Stabilizer (design purpose) & $200(70)$ \\
\hline \hline
\end{tabular}

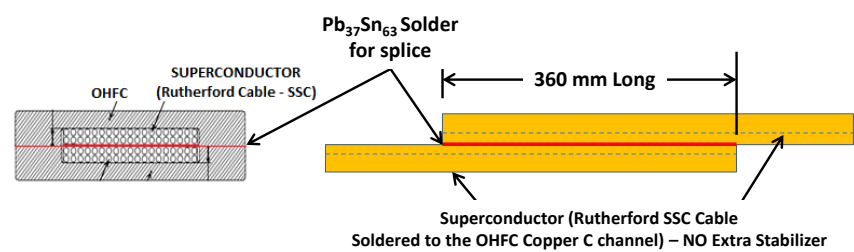

Fig. 3: Typical layout of the test splice in practice for joint resistance evaluation (without any additional copper stabilizer employed in the system),

\section{DESIGN OF CONDUCTOR SPLICES}

a. Key Risk in Joint/Splice Manufacture-The key risk to the joint is high resistance of the solder splice of the cables. High resistance can only come from the lack of even solder distribution and/or the creation of voids and gaps between the cables. This risk is minimized by the qualified fixturing for making splices and by our qualified process discussed later.

b. Solder Details - The soft solder paste used for the splice is $\mathrm{Sn}_{63} \mathrm{~Pb}_{37}$ which is a eutectic mixture which melts totally at $183^{\circ} \mathrm{C}$. The $\mathrm{Sn}_{60} \mathrm{~Pb}_{40}$ solder used to bond the original Rutherford cable into the copper channel has a liquidus of $188^{\circ} \mathrm{C}$ to $190^{\circ} \mathrm{C}$ (melts around above $200^{\circ} \mathrm{C}$ ) so if we control temperature during soldering, the cable's solder will not reflow and the cable will not delaminate out of the copper channel during splice soldering.

c. The splice stability balance - The amount of copper used for conduction and stabilization in a splice for this Torus has to be balanced against the possibility to burn out a conductor if a quench remains localized and un-detected and is not allowed to propagate. Our calculations show that the additional OHFC copper stabilizer bar makes the splice quench tolerant [3]. The bars, extending over the entire splice length, are soldered to the assembly in the same operation that solders the splice.

Specifically, the total amount of copper through the joint zone is enough to allow recovery from a stick slip induced quench because Joule heating falls within the limited cooling power of the conduction cooling. Simultaneously, if there is no recovery the copper volume is small enough to develop a telltale voltage that will trip the fast dump interlocks fast enough to prevent a conductor burn out [4].

d. Electrical isolation to the ground (GND) in vacuum - The insulation system is designed to accommodate for $2.5 \mathrm{kV}$ standoff with polyamide film and a minimum of tracking length $1 / 2$ ". For improved thermal performance, voids in the assembly are potted with two part epoxy blue Stycast $^{\mathrm{TM}} 2850 \mathrm{FT}$. The assembly is Hi-Potted to $1 \mathrm{kV}$ in air to validate electrical isolation and integrity.

\section{Manufacturing OF The TeSt Splices}

a. Design for Manufacturing -An aluminum fixture (Fig. 4) was designed to act as a heat diffuser as well as an alignment jig to firmly hold the stabilized superconducting conductors and extra copper stabilizer elements in place during the soldering process. It employed electric heating elements controlled using thermocouple sensors for precise control of the joint temperature. The fixture was designed compactly to fit in the limited space around the Torus' Cold Beams.. Openings at the edges of the fixture allow the solder splice to be viewed during the heating cycle and any gaps filled with solder. All the individual pieces are initially cleaned to remove oxidation,. Solder paste is then applied to the mating surfaces and the parts assembled in the fixture. A thermocouple monitors the joint temperature during the whole soldering process. The fixture holds the pieces together loosely while the assembly is heated. Once the assembly reaches $183^{\circ} \mathrm{C}$ or the solder is observed to flow, the bar clamps are tightened to force the pieces into intimate contact and to force out excess solder, ensuring a good solder joint.

$b$. The final version of the fixture was designed to include an aluminum plunger bar on top that pushes the splice parts into the slot in the bottom half of the fixture in order to align the pieces and to control where the excess solder flows. These features yielded a final splice within our tolerance as suggested by Fast et al. [5,6]. 


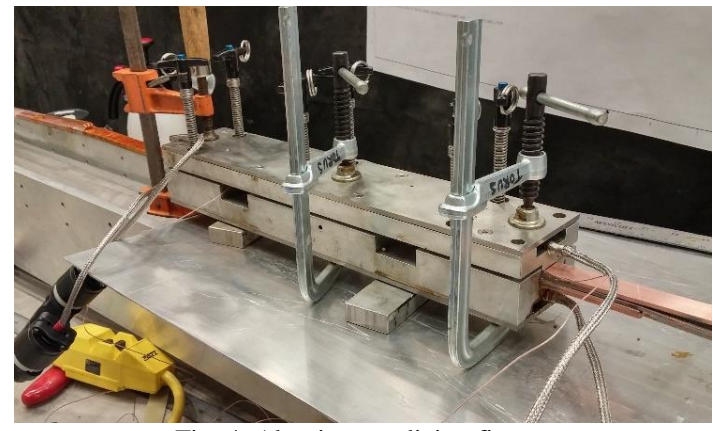

Fig. 4 Aluminum splicing fixture

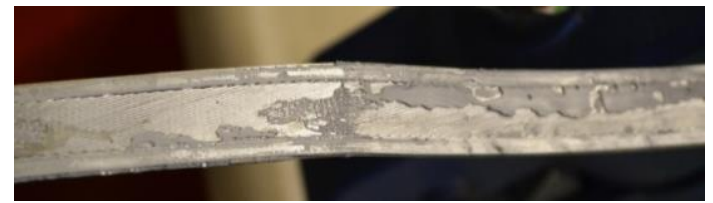

Fig. 5 Conductor after being peeled apart during early splice tests. Note the large areas that solder did not wet. Problems resulted in changes in fixture, flux and technique.

In-house tests - The first 19 test splices were sectioned or peeled apart to look for voids, refining the process and tooling as shown in fig. 5 and 6 . In the end, a repeatable method, able to be performed in the field, was successful. The entire process was captured in a written procedure.

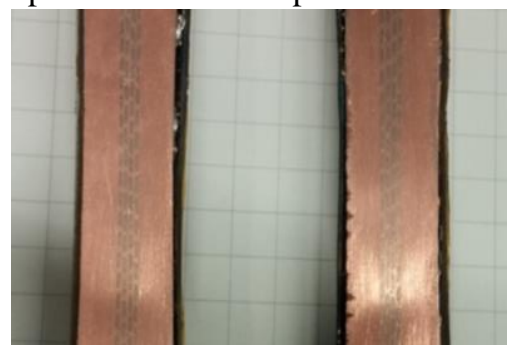

Fig. 6: Splice mock-up end-on view of the sectioned splice cut lengthwise showing void free construction. NbTi strands and solder can be seen in copper matrix. Outer-wrap is Polyamide film and epoxy.

\section{Test RESUlts OF MANUFACTURED TEST SPLICES}

Samples were prepared for critical current $\left(I_{C}\right)$ and n-value measurements and V-I data measured in different magnetic fields in order to validate the data measured on individual strands. Test splices are manufactured at JLab (two conductor made form bare Rutherford Cable soldered to copper channel) of $360 \mathrm{~mm}$ long, similar to splices employed between the two coils in the torus magnet system. The splice prepared at JLab was tested at the University of Durham, UK up to $2000 \mathrm{~A}$ and in one of two $15 \mathrm{~T}$ magnet systems.

\section{a. Critical current $\left(I_{C}\right)$ measurement-}

V-I measurements under an external magnet field generated using Helmholtz coil were carried out in the cable that carry different currents at low electric fields. Reliable $\mathrm{I}_{\mathrm{C}}$ values and $n$-values are obtained at higher E-fields (100 $\mu \mathrm{V} / \mathrm{m}$ ) when the dissipative state ensures the resistance of the superconductor is comparable or higher than the transfer resistances. Standard corrections to magnetic field (self and external magnetic field) and temperature (using scaling laws $[7,8])$ were applied to obtain data at $4.2 \mathrm{~K}$ and integral and half integral values of magnetic field. Typical critical current and n-values are shown in table III for a bare Rutherford cable - sample DR4562.

TABLE III: CRITICAL CURRENT AND N-VALUE DATA FOR DR4562

\begin{tabular}{l|c|c|c}
\hline \multicolumn{5}{|l|}{ Critical Current data } \\
\hline E field Criteria & $10.5 \mathrm{~T}$ & $10.0 \mathrm{~T}$ & $9.5 \mathrm{~T}$ \\
\hline $100 \mu \mathrm{V} / \mathrm{m}$ & $254 \mathrm{~A}$ & $851 \mathrm{~A}$ & $1708 \mathrm{~A}$ \\
$10 \mu \mathrm{V} / \mathrm{m}$ & $165 \mathrm{~A}$ & $656 \mathrm{~A}$ & $1415 \mathrm{~A}$ \\
\hline$n-$ Value & 5 & 9 & 12 \\
\hline $10-100 \mu \mathrm{V} / \mathrm{m}$ & 5
\end{tabular}

b. Joint measurement-

Test set up for splice resistance measurement is carried out using a standard V-I technique integrated with an external magnet field generated using a solenoid and evaluated on sample (DR4621). The sample was formed to be part of the current leads and no additional conductive materials were used in order to ensure joint resistance measurement only. Insulated brass spacers were employed to hold the joint fixed (Fig. 7). $\mathrm{In}_{52} \mathrm{Sn}_{48}$ solder $\left(118^{0} \mathrm{C}\right.$ melting point) was used to solder the voltage taps across the joint of the sample.

The measurements are done at room temperature, LN2 temperature and LHe. At room temperature, the resistance values are predominantly attributable to the resistance of the copper. In preliminary measurements at 3000 A on DR4621, the cable started visibly bubbling and the solder in the channel melting reassuring the measurement and the solder melting point $183^{\circ} \mathrm{C}$. Although the jointed region has twice the crosssectional area of the cable, some melting occurred at the ends of the jointed region. Consistent with these observations, the V-I data in Fig. 8 are not linear at a current higher than about $1000 \mathrm{~A}$. In contrast at $77 \mathrm{~K}$ the data are linear. We attribute the linearity at $77 \mathrm{~K}$ to the joint resistance being about 7 times less (better) than that at room temperature. Thereafter the maximum current for measurements on splices was limited to about $1000 \mathrm{~A}$ and the corresponding V-I traces remained broadly linear up to the maximum current measured as shown in Fig 9.

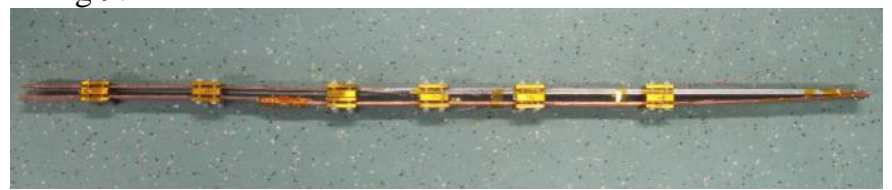

Fig. 7: The sample prepared for joint resistance measurement

V-I measurements on splice sample DR4620 are shown in Figs. $9 \& 10$ are measured at room temperature and $77 \mathrm{~K}$ in zero magnetic field, and at helium temperatures in a $15 \mathrm{~T}$ vertical magnet in Durham. The field profile along the vertical direction of the magnet earlier established using a Hall probe. During the $4.2 \mathrm{~K}$ measurements, the top of the joint was just above the top of the vertical magnet while the bottom of the joint was below the field center. V-I data were obtained from voltage taps across the entire joint.

The resistance of the joint at $4.2 \mathrm{~K}$ saturates at $1.1 \times 10^{-9} \Omega$ in high fields consistent with the entire joint being in the normal state. Fig. 10 shows that when the field at the field center was $3.0 \mathrm{~T}$, the field value was roughly $0.10 \mathrm{~T}$ at the top of the joint. All the solder in the joint was driven into the normal state and the joint reaches its maximum resistance. Subsequent increases in applied field produced little change in the resistance of the joint. The solder at the top of the joint 
became completely normal at a field reasonably close to the $\mathrm{B}_{\mathrm{c} 2}$ of $\mathrm{Pb}-\mathrm{Sn}$ solder (at $5 \mathrm{~K}, \mathrm{~B}_{\mathrm{c} 2} \sim 0.24 \mathrm{~T}$ for $\mathrm{Pb}-\mathrm{Sn}$ solder). In high magnetic fields, when the joint was entirely normal, all V-I traces were reproducible and independent of the history of the applied field. However, when the joint solder was superconducting along part of the length of the joint, the V-I traces were not reversible and intermediate resistances were observed. Typical resistance measured for DR4620 is shown in table IV.

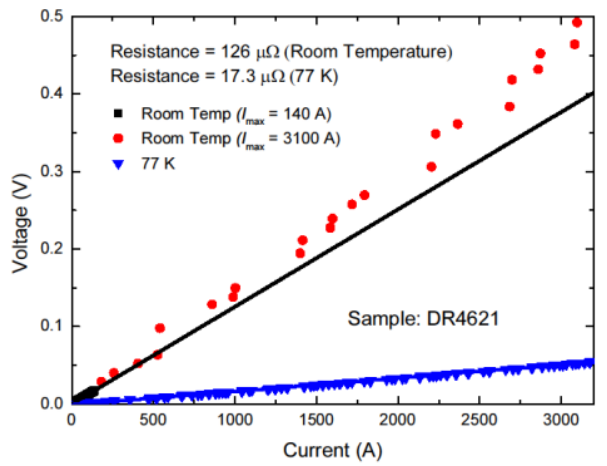

Fig.8: The Voltage vs current at room temperature and $77 \mathrm{~K}$ for the soldered joint of sample DR4621. The black line is an extrapolation of the linear fit from measurements made at room temperature up to 140 A (black squares). The red data were measurements taken up to $3100 \mathrm{~A}$ by which time the solder in the channels was melting. The blue data are from measurements in liquid nitrogen nominally at $77 \mathrm{~K}$.

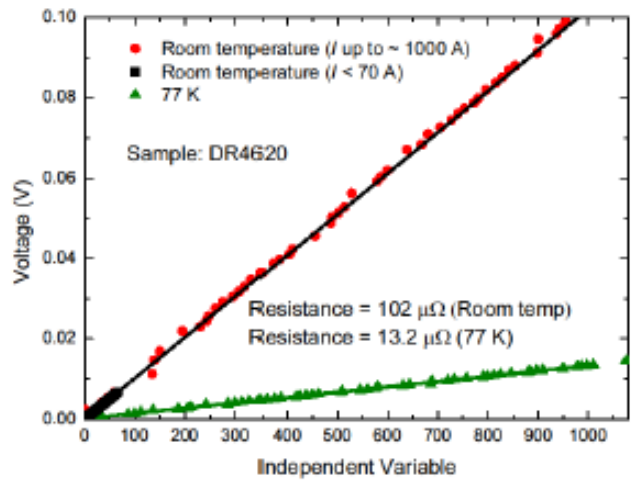

Fig. 9: Voltage vs current at room temperature and $77 \mathrm{~K}$ for the soldered joint of sample DR4620. The black line is an extrapolation of the linear fit from measurements made at room temperature up to $70 \mathrm{~A}$ (black squares). The red data were measurements taken up to $1000 \mathrm{~A}$. The green data are from measurements in liquid nitrogen at nominally at $77 \mathrm{~K}$.

\section{SUMMARY}

1. The procedure and the fixtures for manufacturing the splices between the torus coils has been established and exercised successfully.

2. The resistances across the splices are measured with a vertical magnet capable of producing magnetic flux densities up to $15 \mathrm{~T}$. This resistance measurement includes the magnetoresistance of the conductor and the $\mathrm{Sn}_{63} \mathrm{~Pb}_{37}$ solder in the (completely) normal state.

3. The resistance of the splices measured is about $1.1 \mathrm{n} \Omega$ in LHe $(4.2 \mathrm{~K})$ at elevated magnetic fields, better than the minimum requirement of $7.0 \mathrm{n} \Omega$ (based on $100 \mathrm{~mW}$ joule heating at full operating current).

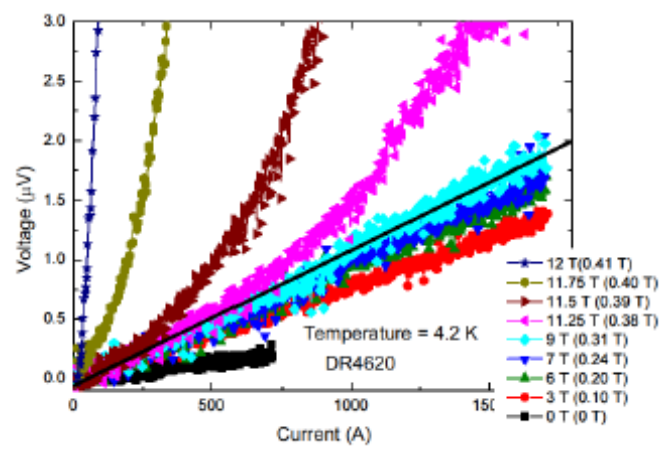

Fig.10: V-I data at different magnetic fields for the soldered joint of sample DR4620 at $4.2 \mathrm{~K}$. The two magnetic field values shown are those at the field center and at the top of the joint (in brackets) which was $279 \mathrm{~mm}$ away from the field center. The black line is a linear fit to the $9 \mathrm{~T}$ data and is equivalent to a resistance of $1.1 \times 10^{-9} \Omega$

TABLE IV: RESISTANCE MEASURED FOR DR4620 AT VARYING MAGNETIC FLUX DENSITY AT $4.2 \mathrm{~K}$

\begin{tabular}{c|c|c|c}
\hline $\begin{array}{c}\text { Joint Length } \\
(\mathrm{mm})\end{array}$ & $\begin{array}{c}\text { Field at field } \\
\text { Centre }(\mathrm{T})\end{array}$ & $\begin{array}{c}\text { Field at the top of } \\
\text { the joint }(\mathrm{T})\end{array}$ & $\begin{array}{c}\text { Joint resistance } \\
\left(\times 10^{-9} \Omega\right)\end{array}$ \\
\hline 404 & 0 & 0 & $\leq 0.1$ \\
& 3 & 0.10 & 0.85 \\
& 6 & 0.20 & 1.0 \\
& 7 & 0.24 & 1.1 \\
& 9 & 0.31 & 1.1 \\
\hline
\end{tabular}

\section{ACKNOWLEDGMENT}

The authors would like to acknowledge the contributions and support of the Cryostat factory team members, technicians, management at JLab and the efforts at the University of Durham, UK for measurement and test. This material is based upon work supported by the U.S. Department of Energy, Office of Science, Office of Nuclear Physics under contract DE-AC05-06OR23177. The U.S. Government retains a nonexclusive, paid-up, irrevocable, world-wide license to publish or reproduce this manuscript for U.S. Government purposes.

\section{REFERENCES}

[1] R. J. Fair, G. L. Young, "Superconducting Magnets for the $12 \mathrm{GeV}$ Upgrade at Jefferson Laboratory", IEEE Trans. on Appl. Supercond., V25 (3), 2015, Digital Object Identifier 10.1109/TASC.2014.2365737

[2] M. Wiseman, et al., "Design and Manufacture of the Conduction Cooled Torus Coils for The Jefferson Laboratory12-GeV Upgrade", IEEE Trans. on Appl. Supercond., V25 (3), 2015, Digital Object Identifier 10.1109/TASC.2014.2376964

[3] P. K. Ghoshal, G. Biallas, R. J. Fair, R. Rajput-Ghoshal, C. Luongo, "Study of Quench Tolerant Stabilized Sections in a Torus Magnet - Coil Lead and Splice for the Jefferson Lab $12 \mathrm{GeV}$ Upgrade," IEEE Trans. on Appl. Supercond., To be submitted, MT24-2015.

[4] P. K. Ghoshal, et.al, "FMEA on the Superconducting Torus for the Jefferson Lab $12 \mathrm{GeV}$ Accelerator Upgrade," IEEE Trans. on Appl. Supercond., V25 (3), 2015, Digital Object Identifier 10.1109/TASC.2015.2388591

[5] R.W. Fast, W.W. Craddock, M. Kobayashi, and M.T. Mruzek, "Electrical and mechanical properties of lead/tin solders and splices for superconducting cables" Cryogenics, 28, 7 (1988)

[6] M. T. Mruzek, "Properties and Methods of Lead/Tin Splices for Superconductors",FermiLab TM-0994, September 1980

[7] L. Bottura and B. Bordini, "JC(B,T,E) Parameterization for the ITER $\mathrm{Nb}_{3} \mathrm{Sn}$ Production," IEEE Transactions on Applied Supercond., V19, 2009, pp. 1521-1524.

M. J. Raine, Y. Tsui, A. Dawson and D. P. Hampshire, Report submitted to Jefferson Lab " Characterization of Rutherford Cables for Jefferson Laboratory", Physics Department, Durham University, Durham, UK (December 2014) 